\title{
Targeted accumulation of selective anticancer depsipeptides by reconstructing the precursor supply in the neoantimycin biosynthetic pathway
}

\author{
Lin Zhou, Yaoyao Shen, Nannan Chen, Wanlu Li, Hou-wen Lin ${ }^{*}$ and Yongjun Zhou* (i)
}

\begin{abstract}
Background: Neoantimycins are a group of 15-membered ring depsipeptides isolated from Streptomycetes with a broad-spectrum of anticancer activities. Neoantimycin biosynthesis is directed by the hybrid multimodular megaenzymes of non-ribosomal peptide synthetase and polyketide synthase. We previously discovered a new neoantimycin analogue unantimycin B, which was demonstrated to have selective anticancer activities and was produced from the neoantimycin biosynthetic pathway with a starter unit of 3-hydroxybenzoate, instead of the 3-formamidosalicylate unit that is common for neoantimycins. However, the low fermentation titre and tough isolation procedure have hindered in-depth pharmacological investigation of unantimycin B as an anticancer agent.

Results: In this work, we genetically constructed two unantimycin B producer strains and inhibited neoantimycins production by removing natO and natJ-L genes essential for 3-formamidosalicylate biosynthesis, therefore facilitating chromatographic separation of unantimycin B from the complex fermentation extract. Based on the $\triangle$ natO mutant, we improved unantimycin B production twofold, reaching approximately $12.8 \mathrm{mg} / \mathrm{L}$, by feeding 3-hydroxybenzoate during fermentation. Furthermore, the production was improved more than sixfold, reaching approximately 40.0 $\mathrm{mg} / \mathrm{L}$, in the $\triangle \mathrm{natO}$ strain introduced with a chorismatase gene highly expressed under a strong promoter for endogenously over-producing 3-hydroxybenzoate.
\end{abstract}

Conclusion: This work provides a case of targeting accumulation and significant production improvement of medicinally interesting natural products via genetic manipulation of precursor biosynthesis in Streptomycetes, the talented producers of pharmaceutical molecules.

Keywords: Anticancer, Depsipeptides, Natural product biosynthesis, 3-Hydroxybenzoate, 3-Formamidosalicylate, Chorismic acid

\section{Introduction}

Streptomyces species are a family of Gram-positive bacteria renowned for their ability to produce a multitude of secondary metabolites with pharmaceutical

\footnotetext{
*Correspondence: franklin67@126.com; zhouyongjun66@163.com Research Center for Marine Drugs, State Key Laboratory of Oncogenes and Related Genes, Department of Pharmacy, Ren Ji Hospital, School of Medicine, Shanghai Jiao Tong University, Shanghai 200127, People's Republic of China
}

activities (Van der Heul et al. 2018). Neoantimycins (NATs) are anticancer compounds of 15-membered ring antimycin-type depsipeptides discovered from the fermentation extract of Streptomycetes (Liu et al. 2016, 2019; Li et al. 2013). The molecular skeleton of NATs was biosynthetically assembled by a hybrid multimodular protein complex of non-ribosomal peptide synthetase (NRPS) and polyketide synthase (PKS), with the starting precursor of 3-formamidosalicylate (3-FAS) (Zhou et al. 2018; Skyrud et al. 2018). We 
previously discovered a new NAT derivative, unantimycin B (UAT-B), from the fermentation extract of terrestrial Streptomyces conglobatus and verified that the biosynthesis of UAT-B was directed by the NAT NRPSPKS with a starter unit of 3-hydroxybenzoate (3-HBA) (Shen et al. 2020) (Fig. 1). UAT-B was demonstrated to process remarkable inhibitory activities against human lung cancer, colorectal cancer, and melanoma cells with no cytotoxic activities towards the corresponding noncancerous cells (Shen et al. 2020). However, UAT-B was produced as a minor product at a yield of 1 to $2 \%$ of the main product (NAT-A) in the wild-type strain. Although the yield level of UAT-B increased relative to that of NAT-A in the heterologous expression host of S. albus J1074 (Shen et al. 2020), the production titre of UAT-B (approximately $6.3 \mathrm{mg} / \mathrm{L}$ ) remained at the same low level as that of the wild-type strain (Additional file 1: Fig. S1). Moreover, the presence of the main product NAT-A seriously interrupted the chromatographic separation of UAT-B. Owing to these challenges, UAT-B had to be prepared with a challenging isolation procedure from the complex fermentation

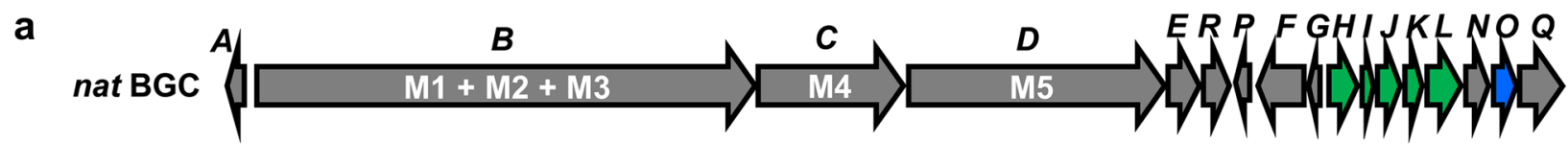

b

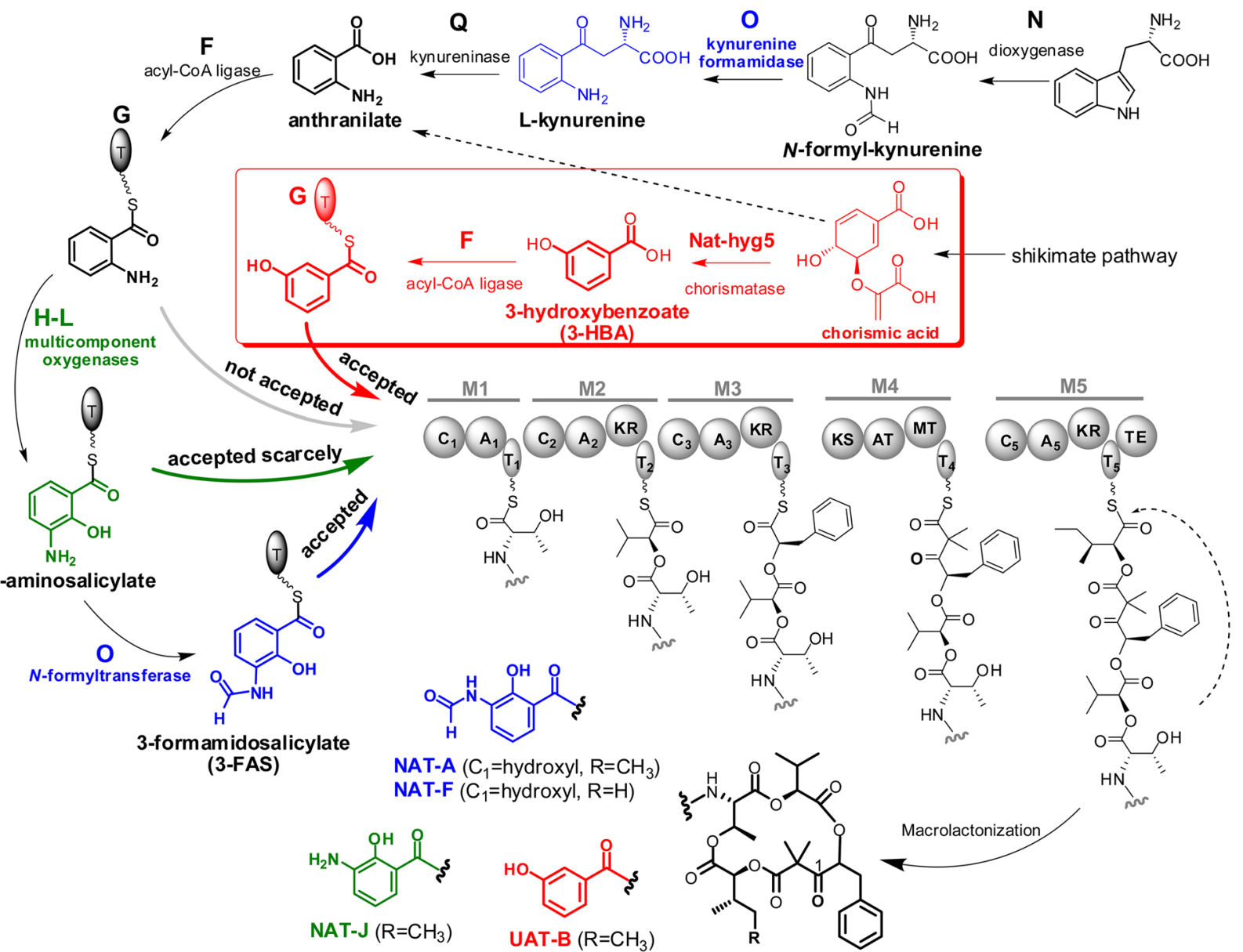

Fig. 1 The gene organization of the NAT biosynthetic gene cluster (nat BGC) (a) and the biosynthetic routes of the starting precursor for the NAT NRPS-PKS assembly line (b). The genes and related products of nat $\mathrm{H}-\mathrm{L}$ and nat $\mathrm{O}$ are marked in green and blue, respectively. The bio-reaction schemes of 3-hydroxybenzoate (3-HBA) generation and loading processes are highlighted in red. The dashed arrow represents that anthranilate could be produced from chorismic acid via primary aromatic amino acid metabolism. A: adenylation; T: thiolation or acyl-carrier protein; C: condensation; KR: ketoreductase; KS: ketosynthase; AT: acyltransferase; MT: methyltransferase; TE: thioesterase 
extract, therefore hindering in-depth pharmacological investigation of the compound as an anticancer agent.

Inspired by the documented approaches of increasing biosynthetic precursor supply and manipulating the substrate specificity of the key enzyme to improve the yields or purity of target metabolites in bacteria (Liu et al. 2018; Fries et al. 2019), we sought to address the above difficulties in this work. Herein, we show the work of successfully directing the NAT NRPS-PKS towards UAT-B production from the original pathway for NATs production by genetically blocking 3-FAS biosynthesis. Based on the resultant mutant with a relatively clear fermentation background, we further achieved significant UAT-B production improvement by enhancing the supply of the starter unit precursor via both exogenous and endogenous strategies. In addition, we obtained some insight for further UAT-B production optimization and investigated the starter unit tolerance of the NAT NRPS-PKS. This work displays a case of targeting accumulation and significant production improvement of medicinally interesting compounds through genetically manipulating precursor supply in the natural product biosynthetic pathway from Streptomycetes, the genius bacterium in producing diverse pharmaceutical molecules.

\section{Materials and methods}

\section{DNA manipulation and chemicals}

The oligonucleotides used in this work were synthesized by Shanghai Generay Biotech Co., Ltd. Restriction endonucleases and T4 DNA ligase were purchased from New England Biolabs. DNA fragment assembly was performed by using the U-Clone master mix kit (Evomic Science, Sunnyvale, CA, USA). Chemicals were purchased from Sigma-Aldrich. Plasmid DNA extraction was performed using a plasmid mini kit (Shanghai Generay Biotech). Genomic DNA used as a PCR template was prepared by using $10 \%$ Chelex 100 resin (Bio-Rad) solution. PCR amplifications were carried out by using Phusion HighFidelity PCR Master Mix (New England Biolabs) for DNA cloning or $2 \times$ FastTaq Master Mix (Shanghai Bioroot Biotech) for colony screening.

\section{Strains, media, and culture conditions}

RJ2 was derived from the terrestrial S. conglobatus and used as a starting producer of UAT-B (Shen et al. 2020). E. coli $\mathrm{DH} 10 \mathrm{~B}$ was used for plasmid construction. $E$. coli ET12567 containing the helper plasmid of pUZ8002 was used as a transitional host for introducing the target plasmid into RJ2. TSBY medium (3\% tryptone soy broth, $0.5 \%$ yeast extract, $10 \%$ sucrose, $0.1 \%$ antifoam) was used to produce mycelium of RJ2. SFM agar medium ( $2 \%$ soya flour, $2 \%$ D-mannitol, $2 \%$ agar) was used for manipulation of conjugation and mutant screening for
RJ2. Fermentation production medium (SGC) consisting of $3 \%$ soybean flour, $5 \%$ glucose, $0.5 \% \mathrm{CaCO}_{3}$, and $0.1 \%$ $(\mathrm{v} / \mathrm{v})$ antifoam was used to produce UAT-B. Streptomyces fermentation was carried out in a $250-\mathrm{mL}$ conical flask fitted with a metal spring, with $1 \%(\mathrm{v} / \mathrm{v})$ inoculation of a 3-day TSBY culture into $50 \mathrm{~mL}$ of SGC medium, and then incubated at $30^{\circ} \mathrm{C}$ and $220 \mathrm{rpm}$ for 5 days. E. coli strains were grown in Luria-Bertani (LB) broth (1\% tryptone, $0.5 \%$ yeast extract, $0.5 \% \mathrm{NaCl})$ or LB agar $(1.5 \%$ agar $)$ at $37^{\circ} \mathrm{C}$ with the corresponding antibiotics supplemented.

\section{Metabolite extraction and HPLC-MS analysis}

To extract fermentation metabolites for HPLC-MS analysis, $7 \mathrm{~mL}$ of 5-day SGC fermentation broth was extracted with an equal volume of ethyl acetate. The extract was dried by vacuum evaporation and re-dissolved in $500 \mu \mathrm{L}$ of methanol. Twenty microlitres of the final sample was injected for HPLC-MS analysis.

HPLC-MS analysis was conducted on a Waters HPLC coupled with a Waters Acquity QDa detector. The analytical HPLC instrument was fitted with a Waters Xbridge C18 column $(250 \mathrm{~mm} \times 4.6 \mathrm{~mm}, 5 \mu \mathrm{m})$. Samples were eluted with the mobile phases of acetonitrile and aqueous $0.1 \%$ formic acid $(\mathrm{v} / \mathrm{v})$ at a flow rate of $0.7 \mathrm{~mL} / \mathrm{min}$ with $80 \%$ of acetonitrile over $30 \mathrm{~min}$ or with a gradient elution of $30-100 \%$ acetonitrile over $30 \mathrm{~min}$. The mass spectrometer was run in positive ionization mode, scanning from $\mathrm{m} / z 200$ to 1250 .

\section{Quantitative analysis of UAT-B production levels}

Quantitative analysis of UAT-B production titres from the fermentation extracts was conducted by using an Agilent 1290 Infinity ultra performance liquid chromatography (UPLC) instrument and an Agilent 6460 triple quadrupole mass spectrometer (QqQ/MS). The UPLC was fitted with a Waters ACQUITY UPLC HSS T3 column $(50 \mathrm{~mm} \times 2.1 \mathrm{~mm}, 1.8 \mu \mathrm{m})$ at $35^{\circ} \mathrm{C}$. A solvent system of acetonitrile and aqueous $0.1 \%$ formic acid ( $/ / \mathrm{v})$ was used for gradient elution with acetonitrile at 60 to $95 \%$ over $4 \mathrm{~min}$ and $95 \%$ for $1 \mathrm{~min}$ at a flow rate of 0.3 $\mathrm{mL} / \mathrm{min}$. The mass spectrometer was run in positive ionization mode with a gas temp $315^{\circ} \mathrm{C}$, a nebulizer pressure of $45 \mathrm{psi}$, a sheath gas temperature of $300{ }^{\circ} \mathrm{C}$, a capillary voltage of $4 \mathrm{kV}$, and a nozzle voltage of $500 \mathrm{~V}$. To produce a standard curve for quantitation, a stock DMSO solution of UAT-B (653 mg/L) was diluted 10, 20, 50, 100, 500 , and 1000 times by using a methanol solution of the fermentation extract from RJ15, a RJ2 derived mutant not exhibiting UAT-B production (Shen et al. 2020). The original data were collected and calculated by Agilent MS workstation software B.08.00. The standard curve was subjected to linear regression with $1 / \mathrm{X}$ weighting. All the samples were analysed in triplicate. 


\section{Fermentation feeding}

DMSO solutions of 3-HBA and L-kynurenine were prepared at concentrations of $1.0 \mathrm{M}$ and $0.5 \mathrm{M}$, respectively. Feeding was carried out by adding $50 \mu \mathrm{L}$ of 3-HBA or 100 $\mu \mathrm{L}$ of $\mathrm{L}-\mathrm{kynurenine}$ to $50 \mathrm{~mL}$ of SGC medium on the $2 \mathrm{nd}$ and 4th days of fermentation, respectively. The broth was extracted for analysis after 5 days of fermentation.

\section{Deletion of the natO gene}

To construct two homologous recombination arms for in-frame deletion of nat $\mathrm{O}$, the primers $\mathrm{L}-\mathrm{O}-\mathrm{S}$ and L-O-A and primers R-O-S and R-O-A (Table 1) were used to amplify a $1499 \mathrm{bp}$ left arm and a $1381 \mathrm{bp}$ right arm, respectively. The two PCR fragments were assembled together by using U-Clone master mix kit with the E. coli-Streptomyces shuttle plasmid pRJ2 linearized with $X b a \mathrm{I}$ and EcoRI (Zhou et al. 2018). The resulting plasmid pRJ65 (Table 2) was transformed into RJ2 via conjugation from a transitional host of $E$. coli ET12567 with the helper plasmid pUZ8002. The target mutant was screened with the primers of C-O-S and C-O-A (Table 1) from the hygromycin-sensitive colonies prepared after two rounds of propagation on SFM plates without antibiotic addition. The expected PCR products were 1021 bp for the target mutation and 1507 bp for RJ2. The PCR products were sequenced to finally confirm the target mutant $\Delta n a t \mathrm{O}$.

\section{Deletion of the natJ-L genes}

To generate a deletion within nat $\mathrm{J}-\mathrm{L}$ genes of the nat $\mathrm{H}-\mathrm{L}$ operon (Fig. 1a), homologous recombination arms were produced by using the primers L-JL-S and L-JL-A for the 1378-bp left arm and the primers R-JL-S and R-JL-A for the 1497-bp right arm (Table 1). The two PCR products were assembled together in $\mathrm{pRJ} 2$ ( $\mathrm{Xba \textrm {I }}$ and EcoRI) by using the same strategy as described above. The resulting plasmid pRJ138 (Table 2) was transformed into RJ2 via conjugation. The target mutant was screened with the

Table 1 PCR primers used in the study

\begin{tabular}{|c|c|}
\hline Primer name & Sequence $\left(5^{\prime}-3^{\prime}\right)$ \\
\hline L-O-S & ATCCCCGGGGACCTGCAGGTCGACTACGACGCCCACTTCTACCTCT \\
\hline $\mathrm{L}-\mathrm{O}-\mathrm{A}$ & ATAGCCGGTGAAGTAGCCGCCGTGGAGCAGCACCACCAACG \\
\hline $\mathrm{R}-\mathrm{O}-\mathrm{S}$ & ACGGCGGCTACTTCACCGGCTATCGGGCCGTGCACAAG \\
\hline $\mathrm{R}-\mathrm{O}-\mathrm{A}$ & TATCACGAGGCCCTTTCGTCTTCAAGAGACGTAGAGCGCGTTGACGCC \\
\hline $\mathrm{C}-\mathrm{O}-\mathrm{S}$ & TCTACGACGAGGTGCTGGGCTTTCTGC \\
\hline $\mathrm{C}-\mathrm{O}-\mathrm{A}$ & AAGAGCTGGACGCTGGTGGAATCGC \\
\hline L-JL-S & ATCCCCGGGGACCTGCAGGTCGACTACGACGTGCTGTGGTCGCTG \\
\hline L-JL-A & AGAGGTAGAAGTGGGCGTCGTCCAGCTCGTCGCTCTCGAACAGCTC \\
\hline$R-J L-S$ & TTCGAGAGCGACGAGCTGGACGACGCCCACTTCTACCTCTG \\
\hline R-JL-A & TATCACGAGGCCCTTTCGTCTTCAAGTGGAGCAGCACCACCAAC \\
\hline$J$ L-T-S & AGTTCCGCAACGTCCTCCTG \\
\hline$J$ L-T-A & AGAACAGCTCGAAACGCACC \\
\hline phiC31-F & TGAGCTCATGAGCGGAGAACGAG \\
\hline phiC31-R & TTTTACAAACTTCTCGACAGACGTAGATCAG \\
\hline puc-F & TACGTCTGTCGAGAAGTTTGTAAAACGACGGCCAGTGCCA \\
\hline puc- $R$ & AGGCGACGGTGTACGCCATATTGATGACATCAGTCGATC \\
\hline hyg-F & ATCGTGCTATGATCGACTGATGTCATCAATATGGCGTACACCGTCGCCTC \\
\hline hyg-R & ATCTCGTTCTCCGCTCATGAGCTCAGGCGCCGGGGGCGGT \\
\hline ermE-pRJ71-S & AAGCTTGGGCTGCAGGTCGACTCTAGTATGCATGCGAGTGTC \\
\hline hyg5-pRJ5-A & AAACAGCTATGACATGATTACGAATTCGATATCAGGCGATGATGCCCTC \\
\hline kasO-hyg5-S & ACAGCGTGCAGGACTGGGGGAGTTATGCTGCGATGCGATTAC \\
\hline SP-hyg5-S & AGAGACAGACCCCCGGAGGTAACCCATATGCTGCGATGCGATTAC \\
\hline pRJ5-S2 & AAGACGTAGCGGCGTAGCGAGAC \\
\hline plB139-A & TGAGTTAGCTCACTCATTAGGCAC \\
\hline AntF-S & TGCGGACCATCGCCGACTACGAGCGGTTCGTC \\
\hline AntF-A & AGCTATGACATGATTACGAATTCGATATCAGGCGCGCAGCGCCTTCTTC \\
\hline NatF-S & GTGCCTGATGTGGCTGACCCAGTTGCTGGAGCGCAAC \\
\hline NatF-A & TGTTGTGTGGAATTGTGAGCGGATAACAATTTCACACAGGAAACAG \\
\hline AACL-A & ATCAGGTCCGCAGCAGTTTCTTGAG \\
\hline
\end{tabular}


Table 2 Bacterial strains and plasmids used in the study

\begin{tabular}{lll}
\hline Strains/plasmids & Application characteristics and antibiotic resistances & Sources/references \\
\hline pRJ2 & E. coli-Streptomyces shuttle plasmid used for constructing gene deletion, hygR, blaR & Zhou et al. (2018) \\
pRJ5 & plB139 derivative containing ermEp* and phiC31 attP site, hygR & This work \\
pRJ65 & pRJ2 derivative used for in-frame deletion of natO, hygR, blaR & This work \\
pRJ138 & pRJ2 derivative used for deletion of natJ-L, hygR, blaR & This work \\
pRJ71 & PCR template used to amplify ermEp*_nat-hyg5 cassette, apramycin and thiostrepton resist- & Shen et al. (2020) \\
& ances & \\
pRJ251 & ermEp*_nat-hyg5 cassette in pRJ5 (Nsil, EcoRV), hygR, phiC31 attP site & This work \\
pRJ252 & kasOp*_nat-hyg5 cassette in pRJ5 (Nsil, EcoRV), hygR, phiC31 attP site & This work \\
pRJ253 & A4p_nat-hyg5 cassette in pRJ5 (Nsil, EcoRV), hygR, phiC31 attP site & This work \\
pRJ255 & kasOp*_antG-natF + A4p_nat-hyg5 in pRJ5 (Nsil, EcoRV), hygR, phiC31 attP site & This work \\
pRJ256 & kasOp*_antGF + A4p_nat-hyg5 in pRJ5 (Nsil, EcoRV), hygR, phiC31 attP site & This work \\
RJ2 & The mutant of S. conglobatus losing conglobatin production & Shen et al. (2020) \\
$\Delta$ natO & In-frame deletion of natO gene from RJ2 & This work \\
$\Delta$ natJ-L & Deletion of natJ-L genes from RJ2 & This work
\end{tabular}

hygR, hygromycin resistance, blaR, ampicillin resistance

primers of JL-T-S and JL-T-A (Table 1) from the hygromycin-sensitive colonies prepared after two rounds of propagation on SFM plates without antibiotic addition. The expected PCR products were $408 \mathrm{bp}$ for the target mutation and $1782 \mathrm{bp}$ for the parent strain RJ2. The PCR products were finally sequenced to confirm the target mutant $\Delta n a t J-\mathrm{L}$.

\section{Construction of nat-hyg5 gene expression cassettes}

The open reading frame (ORF) of the nat-hyg 5 gene combined with different promoters was introduced into the $\Delta n a t \mathrm{O}$ strain via an integrative vector $\mathrm{pRJ} 5$ containing phage phiC31 integration elements (Table 2). pRJ5 was constructed by using a U-Clone master mix kit from three fragments, including the two fragments amplified from pIB139 (Wilkinson et al. 2002) with the primers phiC31-F and phiC31-R for $3241 \mathrm{bp}$ and the primers puc-F and puc- $\mathrm{R}$ for $1536 \mathrm{bp}$, and the $1239 \mathrm{bp}$ fragment of the hygromycin resistance gene amplified with the primers hyg-F and hyg-R from pMS82 (Gregory et al. 2003) (Table 1). The nat-hyg5 gene expression cassettes were constructed with the promoters ermEp* (Wilkinson et al. 2002), kasOp* (Wang et al. 2013), and A4p (Fox and Wang 2008) based on pRJ5 (NsiI, EcoRV) to produce the plasmids pRJ251, pRJ252, and pRJ253, respectively (Table 2). The fragments of $k a s O \mathrm{p} *$ and $A 4 \mathrm{p}$ were ordered from Union-Biotech (Shanghai) Co., Ltd. pRJ251 was generated by assembling pRJ5 (NsiI, EcoRV) and the 1243 bp fragment amplified from pRJ71 (Shen et al. 2020) with the primers ermE-pRJ71-S and hyg5-pRJ5-A (Tables 1 and 2). pRJ252 was assembled from the vector pRJ5 (NsiI, $E c o R V)$ and the two inserts of $214 \mathrm{bp} \mathrm{kasOp*}$, and the $1051 \mathrm{bp}$ fragment amplified from pRJ71 with the primers
kasO-hyg5-S and hyg5-pRJ5-A (Tables 1 and 2). pRJ253 was assembled from the vector pRJ5 (NsiI, EcoRV) and the two inserts of $492 \mathrm{bp} A 4 \mathrm{p}$, and the 1054-bp fragment amplified from pRJ71 with the primers SP-hyg5-S and hyg5-pRJ5-A (Tables 1 and 2). The resultant plasmids were transformed into $\Delta n a t \mathrm{O}$ via conjugation, respectively. The exconjugants were confirmed via the phenotype of hygromycin resistance and colony PCR with the primers pRJ5-S2 and pIB139-A (Table 1).

\section{Construction of antG-natF and antGF gene expression cassettes}

The gene expression cassettes of ant $\mathrm{G}$-nat $\mathrm{F}$ and ant $\mathrm{G}$ ant $\mathrm{F}$ (ant $\mathrm{GF)}$ under the promoter of $\mathrm{kasO \textrm {O }}$ * were introduced into the NsiI site of pRJ253 to give plasmids pRJ255 and pRJ256, respectively (Table 2). The two cassettes were generated by assembling a 426-bp synthesized $\mathrm{kasO \textrm {p }}$ *ant $\mathrm{G}$ fragment with the 1585-bp ant $\mathrm{F}$ and the 1566-bp natF fragments, respectively. The 1585-bp ant $\mathrm{F}$ was amplified with the primers AntF-S and AntF-A from S. albus J1074 (Yan et al. 2012). The 1566-bp natF was amplified with the primers NatF-S and NatF-A from $\mathrm{RJ} 2$. The resulting plasmids were transformed into $\Delta$ nat $\mathrm{O}$ via conjugation. The exconjugants were confirmed by observing hygromycin resistance and colony PCR with the primers pRJ5-S2 and AACL-A (Table 1).

\section{Results and discussion Generation of UAT-B producers with blocked NATs production}

To generate a strain with blocked NAT production that exclusively produced UAT-B, the biosynthesis of 3-FAS was blocked via gene deletion of natO putatively 
involved in both the $\mathrm{N}$-deformylation of $\mathrm{N}$-formylkynurenine and the $\mathrm{N}$-formylation of 3-aminosalicylate or the nat $\mathrm{H}-\mathrm{L}$ genes putatively responsible for epoxidation and a 1,2-shift of the thioester group in 3-FAS biosynthesis (Fig. 1b). To create these mutations, the two E. coli-Streptomyces shuttle plasmids, pRJ65 containing an in-frame deletion (162 out of $219 \mathrm{aa}$ ) in nat $\mathrm{O}$ and pRJ138 containing a deletion (1374 out of $2376 \mathrm{bp}$ ) in the nat J-L gene operon were constructed (Table 2). The expected mutations in $n a t \mathrm{O}$ and nat $\mathrm{J}-\mathrm{L}$ genes were then introduced into the chromosome of the parent strain RJ2 (Shen et al. 2020) through homologous recombination (Fig. 2a). As expected, NAT-A and NAT-F disappeared in $\Delta n a t \mathrm{O}$ and $\Delta n a t \mathrm{~J}-\mathrm{L}$ mutant strains and UAT-B production remained in both mutants according to HPLC-MS analysis of the fermentation extract (Fig. 2b). We originally proposed that UAT-B titre would be higher than that from the parent strain since NAT NRPS-PKS was exclusively utilized to synthesize UAT-B when $3-\mathrm{HBA}$ was unavailable in the mutants. However, the expected increase in UAT-B production was not observed in the $\Delta n a t \mathrm{O}$ or $\Delta n a t \mathrm{~J}-\mathrm{L}$ mutant compared to that in RJ2. The phenotype suggested that insufficient supply of 3-HBA could be a bottleneck for UAT-B production, and supplementation with 3-HBA in fermentation should increase UAT-B production.

\section{Improvement in UAT-B production by feeding 3-HBA in fermentation}

To evaluate whether the 3-HBA supply was a rate-limiting step in improving UAT-B production, a DMSO solution of 3-HBA (1 M) was added twice in a liquid fermentation of the $\Delta n a t \mathrm{O}$ strain to a final concentration of $2 \mathrm{mM}$. HPLC-MS analysis of the resulting fermentation extract indicated that UAT-B production was notably improved compared to the control supplemented with DMSO (Fig. 3). Further quantitative analysis by using UPLC-QqQ/MS confirmed the production

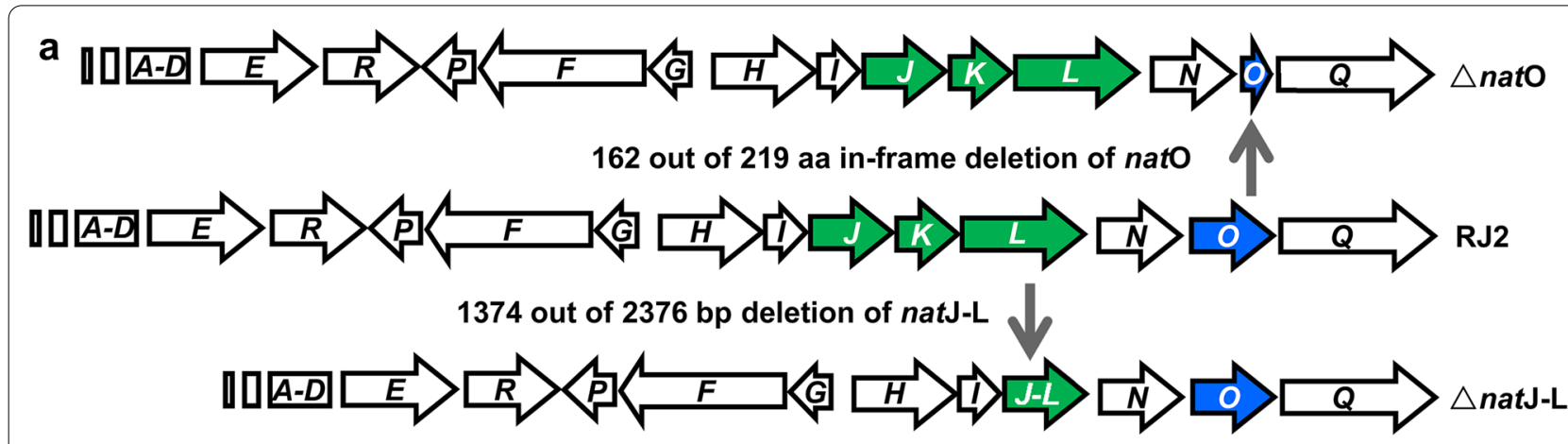

b

\section{UAT-B \& NAT-A}

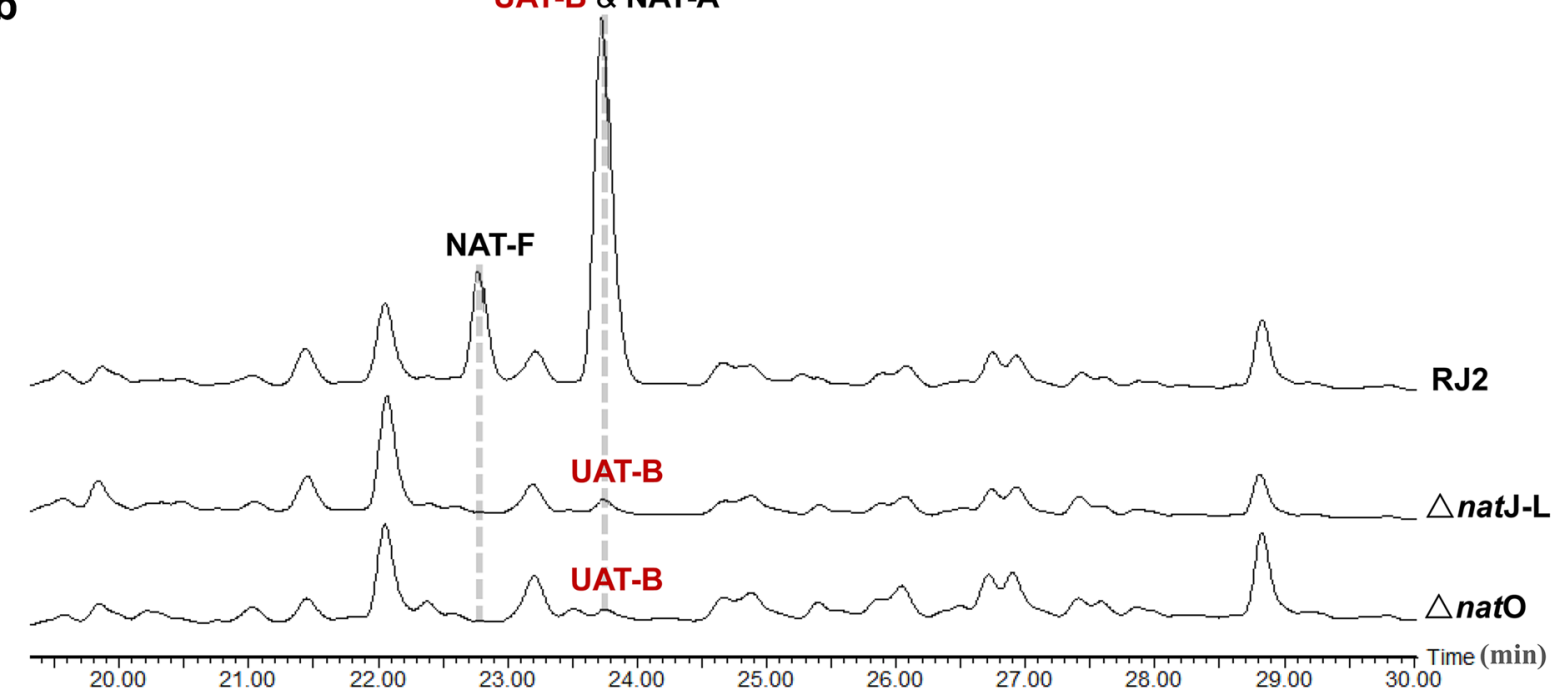

Fig. 2 Schematic diagrams of generating $\triangle$ natO and $\triangle$ natJ-L from RJ2 (a). HPLC analysis of UAT-B, NAT-A and NAT-F from the fermentation extracts of the parent strain RJ2 and the mutants $\triangle$ natO and $\Delta$ natJ-L (b). The data is displayed with UV320 extraction 


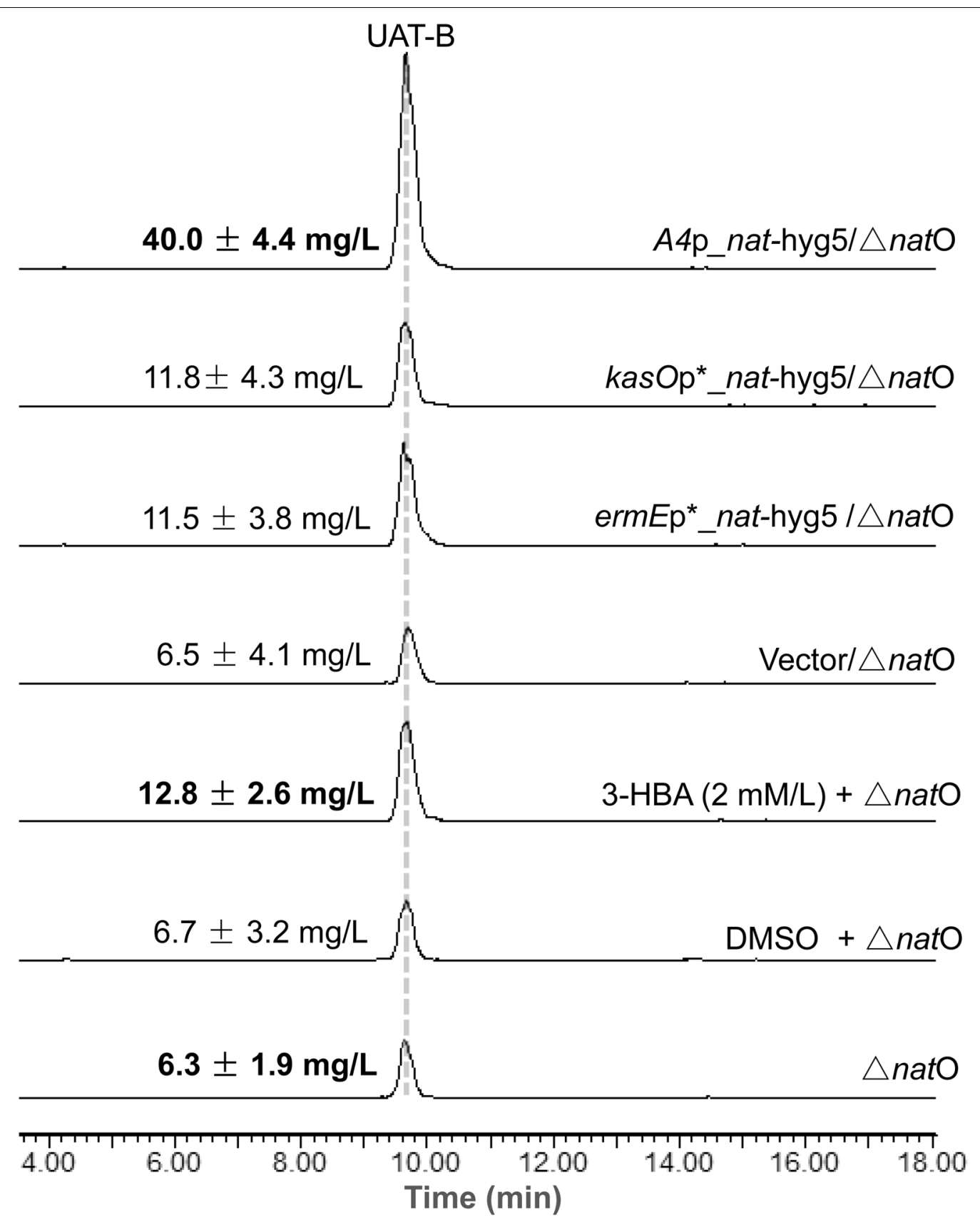

Fig. $3 \mathrm{HPLC}-\mathrm{MS}$ analysis of UAT-B production in the fermentation extracts of the $\triangle$ natO strain fed 3-HBA or containing different nat-hyg3 gene expression cassettes: A4p_nat-hyg5, kasOp*_nat-hyg5 or ermEp*_nat-hyg5. The HPLC-MS data are displayed with the mass extraction of [M+Na] ${ }^{+}$ $\mathrm{m} / \mathrm{z} 676.4$ for UAT-B. The exact UAT-B production titres displayed were obtained by using UPLC-QqQ/MS analysis. All the production titre values shown are the mean \pm standard deviation of triplicate determinations

titre of UAT-B as $12.8 \pm 2.6 \mathrm{mg} / \mathrm{L}$ with 3 -HBA feeding, approximately twice the titre $(6.3 \pm 1.9 \mathrm{mg} / \mathrm{L})$ from fermentation without 3-HBA feeding. The results therefore confirmed our hypothesis that increasing the 3-HBA supply would increase UAT-B production.
Improvement in UAT-B production by overexpressing the gene involved in 3-HBA biosynthesis

To generate a high UAT-B-producing strain independent of the exogenous supply of 3-HBA, the $\Delta$ natO strain was used as a host to overexpress the nat-hyg5 gene, 
which was verified to encode a chorismatase capable of converting endogenous chorismate into 3-HBA in RJ2 (Shen et al. 2020) (Fig. 1b). It is worth mentioning that the $\Delta$ nat J-L strain was not chosen as a host because anthranilate could compete with 3-HBA in the process of being loaded onto an acyl-carrier protein (NatG) by NatF (Fig. 1b). Moreover, considering that heterologous promoters could perform differently in a new host or fermentation medium (Wang et al. 2013), three strong and constitutive promoters were studied, including ermE $\mathrm{p}^{*}$ (Wilkinson et al. 2002), kasOp* (Wang et al. 2013), and A4p (Fox and Wang, 2008). Therefore, the gene expression cassettes of ermEp*_nat-hyg5, kasOp*_nat-hyg5, and A4p_nat-hyg5 were constructed based on the phiC31 integrative vector pRJ5. The resulted plasmids pRJ251, pRJ252, and pRJ253 (Table 2) were conjugated into the $\Delta$ natO strain, respectively. According to HPLC-MS analysis, significant UAT-B production improvement was obtained upon each of the three nat-hyg5 cassettes compared to the empty vector control (Fig. 3). Among the three cassettes, A4p_nat-hyg5 gave the highest production of UAT-B as determined by using UPLC-QqQ/MS analysis, giving a titre of $40.0 \pm 4.4 \mathrm{mg} / \mathrm{L}$, more than three times that achieved by feeding 3-HBA and more than six times the titre of the starting $\Delta n a t \mathrm{O}$ strain, $6.3 \pm 1.9$ mg/L (Fig. 3).

\section{Investigation of UAT-B production by overexpressing the genes involved in loading the starter unit}

Considering another possibility that UAT-B production could be limited at the stage of loading the starting precursor, in which acyl-CoA ligase (NatF) authentically loaded anthranilate, rather than 3-HBA, onto the acylcarrier protein (NatG) in the 3-FAS biosynthetic pathway (Fig. 1b), we decided to further optimize UAT-B production by raising the expression levels of NatG and NatF based on the host of the $\Delta n a t \mathrm{O}$ strain. As documented, natGF genes could be functionally replaced by the homologous genes of antGF, which were derived from the antimycin biosynthetic gene cluster and showed $80-90 \%$ identity to $n a t$ GF in the protein sequence (Zhou et al. 2018). Moreover, AntF (80\% identity to NatF) was demonstrated to have a wide range of substrate specificities (Yan et al. 2012; Sandy et al. 2012), encouraging us to investigate whether AntF was more tolerant than NatF in loading 3-HBA onto the acyl-carrier protein (AntG). We therefore designed two plasmids to co-express the nat-hyg 5 gene with the antG-nat $\mathrm{F}$ and ant $\mathrm{G}$-ant $\mathrm{F}$ (ant $\mathrm{GF}$ ) cassettes, respectively, to compare the contributions of $n a t \mathrm{~F}$ and ant $\mathrm{F}$ to UAT-B production improvement. Finally, the cassettes of kasOp*antG-nat $\mathrm{F}$ and $\mathrm{kasOp} *$ antGF were introduced into the plasmid pRJ253 containing A4p_nat-hyg5 to generate pRJ255 and
pRJ256, respectively. However, neither of the cassettes increased UAT-B production in the $\Delta n a t \mathrm{O}$ strain, as determined by HPLC-MS analysis (Additional file 1: Fig. S2). We hypothesized that an alternative rate-limiting step could be the first condensation stage of NAT NRPSPKS, in which the condensation domain $\left(C_{1}\right)$ formed an amide bound between the starter unit, e.g., 3-HBA or 3-FAS, tethered on NatG and the threonine loaded on the thiolation domain (T1). It was therefore possible to further improve UAT-B production by increasing the preference of $\mathrm{C}_{1}$ towards 3-HBA. Moreover, tuning the substrate specificity of NatF (acyl-CoA ligase) towards 3 -HBA through site-mutation could be another candidate strategy.

\section{Investigation of the starter unit tolerance of NAT NRPS-PKS by analysing the metabolites from $\Delta$ natO and $\Delta$ natJ-L mutants}

The structural features of reported NAT analogues suggest that NAT NRPS-PKS can accept diverse starting precursors, such as benzoic acid, 3-HBA, and 3-aminosalicylic acid, except 3-FAS (Izumikawa et al. 2007; Lim et al. 2016; Lin et al. 2019). Taking advantage of the $\Delta$ natJ-L mutant generated in the work, we explored whether NAT NRPS-PKS could accept anthranilate as a starter unit. According to the proposed 3-FAS biosynthesis, nat $\mathrm{H}-\mathrm{L}$ encodes the multicomponent oxygenase catalysing epoxidation and a 1,2-shift of the thioester group in anthraniloyl-S-NatG to produce 3-aminosalicyloyl$S$-NatG (highlighted in green, Fig. 1b) (Schoenian et al. 2012; Liu et al. 2015); thus, disruption of nat H-L would accumulate anthranilate as a starter unit to produce new NAT analogues. However, no expected products were detected in the fermentation extract of $\Delta n a t$ J-L by using HPLC-MS, suggesting that anthranilate did not result in a product in NAT NRPS-PKS. Moreover, by using the nat $\mathrm{O}$ mutant, we evaluated whether 3-aminosalicylate could be more efficiently loaded as a starter unit to produce NAT-J, a minor product putatively derived from the NAT biosynthetic pathway (Lin et al. 2019). In the proposed 3-FAS biosynthesis, NatO, showing $76 \%$ identity to the AntO derived from the antimycin biosynthetic pathway, was believed to work as a difunctional enzyme conducting two catalytic processes: the deformylation of $\mathrm{N}$-formyl-kynurenine to generate L-kynurenine and the $\mathrm{N}$-formylation of 3-aminosalicylate to produce 3-FAS (highlighted in blue, Fig. 1b) (Liu et al. 2015; Zhou et al. 2018). Thus, $\Delta$ natO should lose NAT-J production due to the destroyed supply of L-kynurenine. However, NAT-J production in $\Delta n a t \mathrm{O}$ was still observed at a similar level as that in the parent strain, suggesting that the blocked L-kynurenine could be complemented, for instance, by the anthranilate produced from primary aromatic amino 
acid metabolism (Sprenger 2007) (Fig. 1b). Moreover, because NatQ, NatF, NatG, and NatH-L worked efficiently in NAT-A biosynthesis, we hypothesized that exogenously supplemented L-kynurenine could be processed sufficiently to deliver 3-aminosalicylate for NAT-J production (Fig. 1b). However, supplementation with 2 $\mathrm{mM}$ L-kynurenine did not increase NAT-J production in the $\Delta$ nat $\mathrm{O}$ strain according to HPLC-MS analysis (Fig. 4), indicating that 3-aminosalicylate could only be incorporated as a starter unit by NAT NRPS-PKS to a very low extent.

\section{Conclusions}

NATs are a group of 15 -membered ring depsipeptides with a broad spectrum of anticancer activities (Liu et al. 2016, 2019). NAT biosynthesis is directed by a hybrid multimodular protein complex of non-ribosomal peptide synthetase (NRPS) and polyketide synthase (PKS) with the starting precursor of 3-formamidosalicylate (3-FAS) (Zhou et al. 2018; Skyrud et al. 2018). We previously discovered the new NAT derivative unantimycin B (UAT-B), which exhibited selective anticancer activities and was verified to be produced by NAT NRPS-PKS with a starter unit of 3-hydroxybenzoate (3-HBA) rather than 3-FAS (Shen et al. 2020) (Fig. 1b). However, UAT-B was produced in low yield (approximately $6.3 \pm 1.9 \mathrm{mg} / \mathrm{L}$ ), and it was difficult to chromatographically separate UAT-B from the dominant product NAT-A in the complex fermentation extract (Fig. 2b), therefore hindering in-depth pharmacological investigation of the compound as an anticancer agent. In this work we blocked NAT-A and NAT-F production via deletion of nat $\mathrm{O}$ or natJ-L genes essential to 3-FAS biosynthesis to direct NAT NRPS-PKS to exclusively produce UAT-B (Figs. 1 and 2). However, the expected production improvement in UAT-B was not observed in the mutants, implying that insufficient supply of 3-HBA could be a bottleneck for improving UAT-B production. This proposal was then supported by the results that feeding $3-\mathrm{HBA}$ to the $\Delta n a t \mathrm{O}$ mutant improved UAT-B production more than twofold. Next, we generated a strain capable of producing 3-HBA

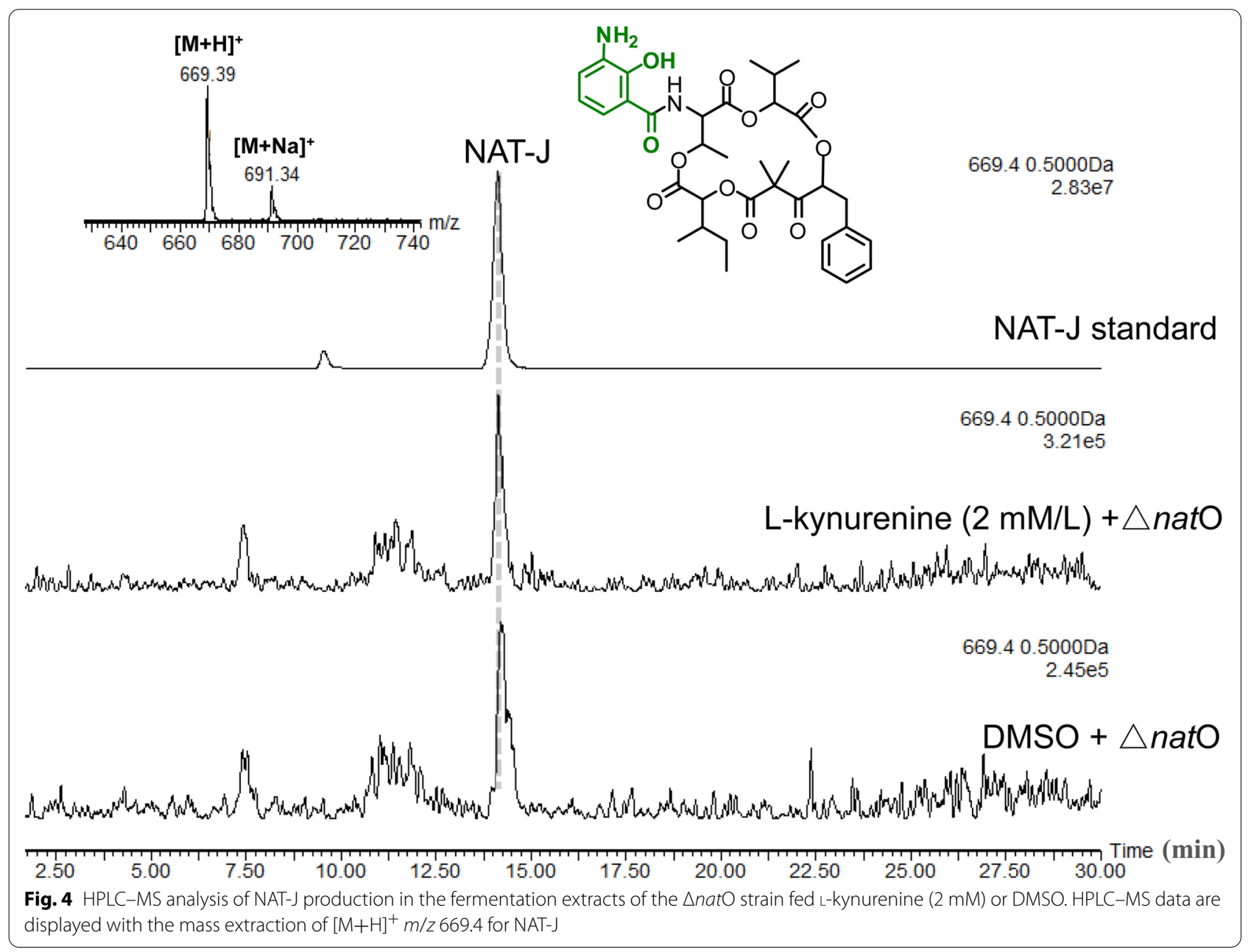


endogenously by over-expressing a chorismatase gene involved in 3-HBA biosynthesis and further increased UAT-B production more than sixfold, reaching $40.0 \pm 4.4 \mathrm{mg} / \mathrm{L}$ (Fig. 3). Moreover, by careful analysis of the metabolites of the $\Delta n a t \mathrm{O}$ and $\Delta n a t$ J-L mutants, we proposed that NAT NRPS-PKS could incorporate 3 -aminosalicylate to a low extent and would not recognize anthranilate as a starter unit (Fig. 1b). In summary, this work shows a case of targeted accumulation and significant production improvement of a medicinally interesting natural product by genetically manipulating the precursor supply in Streptomycetes, the talented industrial producers of bioactive molecules.

\section{Abbreviations}

HPLC-MS: High-performance liquid chromatography-mass spectrum; UPLC: Ultra performance liquid chromatography; QqQ-MS: Triple quadrupole mass spectrometry; DMSO: Dimethyl sulfoxide.

\section{Supplementary Information}

The online version contains supplementary material available at https://doi. org/10.1186/s40643-021-00397-z.

Additional file 1: Fig. S1. HPLC-MS analysis of the UAT-B production leve from the reported strain pRJ71+pRJ4/J1074, which was generated as the heterologous expression system for producing UAT-B and NATs (Shen et al. 2020). The mutant strain $\triangle$ natO generated in the work was used as control. The data are displayed with the mass extraction of $\mathrm{m} / \mathrm{z} 676.4$, $[\mathrm{M}+\mathrm{Na}]^{+}$for UAT-B. Fig. S2. HPLC-MS analysis of the UAT-B production levels from the $\triangle$ natO derivative strains, which individually contained the plasmids pRJ253 (A4p_nat-hyg5), pRJ255 (kasOp*_antG-natF + A4p_nathyg5), and pRJ256 (kasOp*_antG-antF + A4p_nat-hyg5). The empty vector pRJ5 was used as negative control. The data are displayed with the mass extraction of $\mathrm{m} / \mathrm{z}$ 676.4, $[\mathrm{M}+\mathrm{Na}]^{+}$for UAT-B.

\section{Acknowledgements}

We thank Prof. Xinyi He from School of Life Sciences and Biotechnology, Shanghai Jiao Tong University for kindly providing pMS82. Dr. Yongjun Zhou gratefully acknowledges Prof. Peter F. Leadlay from University of Cambridge for providing the starting strain S. conglobatus in the research.

\section{Authors' contributions}

LZ, YYS, NNC, and WLL did the experiments. HWL provided resources. YJZ supervised the project, designed the experiments, and wrote the manuscript. All authors read and approved the final manuscript.

\section{Funding}

This work received financial support from the National Science Foundation of China (Nos. 31670096, 32070070, and 31929001) and the Innovative Re-search Team of High-Level Local Universities in Shanghai.

\section{Availability of data and materials}

Not applicable.

\section{Declarations}

\section{Ethics approval and consent to participate}

Not applicable.

\section{Consent for publication}

All authors have read this article and have approved its submission to Bioresources and Bioprocessing

\section{Competing interests}

The authors declare that they have no competing interests.

Received: 24 February 2021 Accepted: 11 May 2021

Published online: 22 May 2021

\section{References}

Fox B P, Wang H (2008) Expression of streptomyces subtilisin inhibitor (SSI) proteins in Bacillus and Streptomyces sp. Patent WO2009096916, Publication: 29 Jan 2008

Fries A, Mazzaferro LS, Grüning B, Bisel P, Stibal K, Buchholz PCF, Pleiss J, Sprenger GA, Müller M (2019) Alteration of the route to menaquinone towards isochorismate-derived metabolites. ChemBioChem 20(13):1672-1677

Gregory MA, Till R, Smith MC (2003) Integration site for Streptomyces phage phiBT1 and development of site-specific integrating vectors. J Bacteriol 185(17):5320-5323

Izumikawa M, Ueda JY, Chijiwa S, Takagi M, Shin-ya K (2007) Novel GRP78 molecular chaperone expression down-regulators JBIR-04 and -05 isolated from Streptomyces violaceoniger. J Antibiot (tokyo) 60(10):640-644

Li X, Zvanych R, Vanner SA, Wang W, Magarvey NA (2013) Chemical variation from the neoantimycin depsipeptide assembly line. Bioorg Med Chem Lett 23(18):5123-5127

Lim CL, Nogawa T, Okano A, Futamura Y, Kawatani M, Takahashi S, Ibrahim D, Osada H (2016) Unantimycin A, a new neoantimycin analog isolated from a microbial metabolite fraction library. J Antibiot (tokyo) 69(6):456-458

Lin X, Zhou Y, Liu L, Zhu H, Chen Y, Wang S, Sun F, Chai L, Liu B, Xu S, Lin HW (2019) Compound discovery and structure-activity relationship study of neoantimycins against drug-resistant cancer cells. Front Chem 7:481

Liu J, Zhu X, Seipke RF, Zhang W (2015) Biosynthesis of antimycins with a reconstituted 3-formamidosalicylate pharmacophore in Escherichia coli. ACS Synth Biol 4(5):559-565

Liu J, Zhu X, Kim SJ, Zhang W (2016) Antimycin-type depsipeptides: discovery, biosynthesis, chemical synthesis, and bioactivities. Nat Prod Rep 33(10):1146-1165

Liu R, Deng Z, Liu T (2018) Streptomyces species: ideal chassis for natural product discovery and overproduction. Metab Eng 50:74-84

Liu L, Zhu H, Wu W, Shen Y, Lin X, Wu Y, Liu L, Tang J, Zhou Y, Sun F, Lin HW (2019) Neoantimycin F, a streptomyces-derived natural product induces mitochondria-related apoptotic death in human non-small cell lung cancer cells. Front Pharmacol 10:1042

Sandy M, Rui Z, Gallagher J, Zhang W (2012) Enzymatic synthesis of dilactone scaffold of antimycins. ACS Chem Biol 7(12):1956-1961

Schoenian I, Paetz C, Dickschat JS, Aigle B, Leblond P, Spiteller D (2012) An unprecedented 1,2-shift in the biosynthesis of the 3-aminosalicylate moiety of antimycins. ChemBioChem 13(6):769-773

Shen Y, Sun F, Zhang L, Cheng Y, Zhu H, Wang SP, Jiao WH, Leadlay PF, Zhou Y, Lin HW (2020) Biosynthesis of depsipeptides with a 3-hydroxybenzoate moiety and selective anticancer activities involves a chorismatase. J Biol Chem 295(16):5509-5518

Skyrud W, Liu J, Thankachan D, Cabrera M, Seipke RF, Zhang W (2018) Biosynthesis of the 15-membered ring depsipeptide neoantimycin. ACS Chem Biol 13(5):1398-1406

Sprenger GA (2007) From scratch to value: engineering Escherichia coli wild type cells to the production of L-phenylalanine and other fine chemicals derived from chorismate. Appl Microbiol Biotechnol 75(4):739-749

Van der Heul HU, Bilyk BL, McDowall KJ, Seipke RF, Van Wezel GP (2018) Regulation of antibiotic production in Actinobacteria: new perspectives from the post-genomic era. Nat Prod Rep 35(6):575-604

Wang W, Li X, Wang J, Xiang S, Feng X, Yang K (2013) An engineered strong promoter for streptomycetes. Appl Environ Microbiol 79(14):4484-4492

Wilkinson CJ, Hughes-Thomas ZA, Martin CJ, Böhm I, Mironenko T, Deacon M, Wheatcroft M, Wirtz G, Staunton J, Leadlay PF (2002) Increasing the efficiency of heterologous promoters in actinomycetes. J Mol Microbiol Biotechnol 4(4):417-426 
Yan Y, Zhang L, Ito T, Qu X, Asakawa Y, Awakawa T, Abe I, Liu W (2012) Biosynthetic pathway for high structural diversity of a common dilactone core in antimycin production. Org Lett 14(16):4142-4145

Zhou Y, Lin X, Williams SR, Liu L, Shen Y, Wang SP, Sun F, Xu S, Deng H, Leadlay PF, Lin HW (2018) Directed accumulation of anticancer depsipeptides by characterization of neoantimycins biosynthetic pathway and an NADPHdependent reductase. ACS Chem Biol 13(8):2153-2160

\section{Publisher's Note}

Springer Nature remains neutral with regard to jurisdictional claims in published maps and institutional affiliations.

\section{Submit your manuscript to a SpringerOpen ${ }^{\circ}$ journal and benefit from:}

- Convenient online submission

- Rigorous peer review

- Open access: articles freely available online

- High visibility within the field

- Retaining the copyright to your article

Submit your next manuscript at $\boldsymbol{\nabla}$ springeropen.com 